\title{
BMJ Open Knee moments of anterior cruciate ligament reconstructed and control participants during normal and inclined walking
}

\author{
Raghav K Varma, Lynsey D Duffell, Dinesh Nathwani, Alison H McGregor
}

To cite: Varma RK, Duffell LD, Nathwani D, et al. Knee moments of anterior cruciate ligament reconstructed and control participants during normal and inclined walking. BMJ Open 2014;4:e004753. doi:10.1136/bmjopen-2013004753

- Prepublication history for this paper is available online. To view these files please visit the journal online (http://dx.doi.org/10.1136/ bmjopen-2013-004753)

Received 24 December 2013 Revised 25 April 2014 Accepted 16 May 2014

CrossMark

MSK Lab, Imperial College London, London, UK

Correspondence to Professor Alison McGregor; a.mcgregor@imperial.ac.uk

\section{ABSTRACT}

Objectives: Prior injury to the knee, particularly anterior cruciate ligament (ACL) injury, is known to predispose one to premature osteoarthritis $(O A)$. The study sought to explore if there was a biomechanical rationale for this process by investigating changes in external knee moments between people with a history of ACL injury and uninjured participants during walking: (1) on different surface inclines and (2) at different speeds. In addition we assessed functional differences between the groups.

Participants: 12 participants who had undergone ACL reconstruction (ACLR) and 12 volunteers with no history of knee trauma or injury were recruited into this study. Peak knee flexion and adduction moments were assessed during flat (normal and slow speed), uphill and downhill walking using an inclined walkway with an embedded Kistler Force plate, and a ten-camera Vicon motion capture system. Knee injury and Osteoarthritis Outcome Score (KOOS) was used to assess function. Multivariate analysis of variance (MANOVA) was used to examine statistical differences in gait and KOOS outcomes.

Results: No significant difference was observed in the peak knee adduction moment between ACLR and control participants, however, in further analysis, MANOVA revealed that ACLR participants with an additional meniscal tear or collateral ligament damage (7 participants) had a significantly higher adduction moment $(0.33 \pm 0.12 \mathrm{Nm} / \mathrm{kg} \mathrm{m})$ when compared with those with isolated ACLR (5 participants, $0.1 \pm 0.057 \mathrm{Nm} / \mathrm{kg} \mathrm{m}$ ) during gait at their normal speed $(p<0.05)$. A similar (nonsignificant) trend was seen during slow, uphill and downhill gait.

Conclusions: Participants with an isolated ACLR had a reduced adductor moment rather an increased moment, thus questioning prior theories on $\mathrm{OA}$ development. In contrast, those participants who had sustained associated trauma to other key knee structures were observed to have an increased adduction moment. Additional injury concurrent with an ACL rupture may lead to a higher predisposition to osteoarthritis than isolated $\mathrm{ACL}$ deficiency alone.

\section{Strengths and limitations of this study}

- To the best of our knowledge, this is the first report looking at external moments during inclined and declined walking for anterior cruciate ligament (ACL) reconstruction participants.

- In addition to looking into the external moments of the affected and unaffected knee, this study also looked at the effect of gait speed on external moments and differences in Knee injury and Osteoarthritis Outcome Score (KOOS) for each group.

- This study provides a potential explanation for the disparity seen in previous studies looking into peak knee adduction moment in ACL reconstruction (ACLR) and matched control participants.

- This study suggests that injuries to other key knee structures may play a bigger part in inducing osteoarthritis than ACL injury alone.

- One limitation to this study is the small sample size, in particular after dividing our ACLR group into ACLR+ and ACLR-groups.

\section{INTRODUCTION}

Anterior cruciate ligament (ACL) injuries are common, exceeding 100000 annual cases in the USA. ${ }^{1}$ The majority are sports-related injuries, and lead to knee instability as a result of increased anterior tibial translation and anterolateral rotation. ${ }^{2}$ ACL reconstruction (ACLR) is the primary treatment for an ACL rupture and permits return to a range of high-level activities including sport. It is accepted that people with ACL injuries, including those who undergo surgical reconstruction, are prone to further knee degeneration $^{3}$ and early osteoarthritis (OA). ${ }^{4} \quad 5$ Lohmander et $a \hat{l}$ reviewed 127 publications and determined that the overall mean incidence of developing OA after an ACL injury with/without reconstruction is over $50 \%^{3}$ with the majority noting radiographic signs of OA 10 years after injury. Gait biomechanics 
are considered to play a vital part in knee joint degeneration, ${ }^{5}{ }^{6}$ with altered kinematics and kinetics changing the distribution of mechanical load on the knee. ${ }^{7}$ This in turn is postulated to lead to cartilage wear ${ }^{5-7}$ and eventually knee osteoarthritis.

There is consensus among researchers that ACL deficient patients employ different gait strategies. ${ }^{8}$ In vivo studies have found reduced knee flexion, ${ }^{5}$ increased internal tibial rotation ${ }^{5}$ and increased knee adduction moment ${ }^{10}$ during level walking to be the three main changes in external knee moments following an ACL rupture. Furthermore, research has indicated that ACL reconstruction does not restore normal knee mechanics. ${ }^{11}$ Berchuck et $a l^{12}$ in 1990 noted reduced knee flexion during normal gait, indicating a coping strategy termed quadriceps avoidance gait. Anterior displacement of tibia through the contraction of quadriceps is balanced by the ACL when the knee is at an angle of $0-45^{\circ} .{ }^{12}$ People with ACL rupture and/or ACLR are found to have quadriceps activation deficits, ${ }^{13}$ which may be due to a central regulatory mechanism to avoid further joint damage by these muscle groups. Gait adaptations in the sagittal plane can lead to knee joint instability and ligament laxity. ${ }^{14}$ This may result in osteoarthritis initiation and progression. ${ }^{14}$

High moments in frontal and transverse planes of the knee have been linked to OA. ${ }^{5}$ ACLR has been shown to restore rotational stability, ${ }^{9}{ }^{15}$ however high-knee adduction moments (KAM) after reconstruction have been observed $^{16}$ but such changes are not universally agreed. ${ }^{17} 18$ This is of particular importance since a $1 \%$ increase in adduction moment at the knee is thought to increase the risk of knee OA by 6.5 times. ${ }^{19}$ The discrepancies in previous studies may be due to different walking speeds, and higher KAM may only be evident during more challenging tasks. Hence in this study we aimed to gain a better understanding of peak knee moments in the frontal and sagittal planes during gait at different speeds and inclines. Our primary aim is to compare peak knee moments in the sagittal and frontal planes of ACLR participants with healthy controls on sloped surfaces, with a view to explore the biomechanical basis for the observation that ACL injury predisposes one to OA. Our secondary aim was to investigate the effect of speed on peak moments. Finally, we compared functional outcome scores between groups using the Knee injury and Osteoarthritis Outcome Score (KOOS).

\section{METHODS}

This cross-sectional study explored peak knee moments between ACLR and healthy control participants during inclined walking. The study was approved by the Imperial College Research Ethics Committee. We used the Strengthening the Reporting of Observational studies in Epidemiology statement as a checklist for our observational study. ${ }^{20}$

A total number of 24 participants participated in this study and written informed consent was obtained; details are provided in table 1 . The ACLR inclusion criteria were: aged between 18 and 60 years; body mass index (BMI) $<30 \mathrm{~kg} / \mathrm{m}^{2}$; a complete, unilateral ACL rupture followed by a single bundle hamstring autograft reconstruction that was performed at least 1 year ago with no history of knee trauma or injury to their contralateral leg. Participants who were unable to walk comfortably on a $10^{\circ}$ incline walkway were also excluded. The control group did not have any muscular or neurological lower limb pathology and were matched to the ACLR participants with respect to gender, activity, height, weight and their dominant leg (leg preference for kicking). All particpants completed the KOOS. ${ }^{21}$ We measured the participants' activity levels using Tegner activity scale. ${ }^{22}$

A three-dimensional motion analysis system (Vicon MX T-20 System, Vicon, Oxford, UK) was used to collect kinematic data for normal, slow, upslope and downslope gait. This software used ten-motion capture cameras to pick up 35 reflective markers at a sampling rate of $100 \mathrm{~Hz}$. The reflective markers were placed bilaterally on the head of the second metatarsal, head of the fifth metatarsal, head of talus, calcaneal tuberosity, medial and lateral malleolus, medial and lateral femoral epicondyle, anterior superior iliac spine, posterior superior iliac spine, acromion and one single marker on the manubrium. Marker clusters (3 reflective markers on each) were affixed bilaterally to the calf and thigh. Kinetic data (ground reaction force) were collected using portable force plates (Kistler Instruments AG, Winterthur, Switzerland) at a sampling rate of $1000 \mathrm{~Hz}$ and were synchronised with the camera data.

The participants were asked to walk barefoot. A $5 \mathrm{~min}$ self-directed warm up allowed the participants to familiarise themselves with each task.

A $7 \mathrm{~m}$ long walkway was used, 2.5 metres of which could be raised to form a ramp, at an incline of $10^{\circ}$. It

Table 1 Participant characteristics, activity level and time since surgery

\begin{tabular}{lccc}
\hline & ACLR (SD) & Control (SD) & Unpaired t test \\
\hline Age (year) & $30.5(8.68)$ & $24.8(8.81)$ & $\mathrm{p}=0.125$ \\
Height $(\mathrm{m})$ & $1.76(0.13)$ & $1.73(0.11)$ & $\mathrm{p}=0.547$ \\
Weight $(\mathrm{kg})$ & $75(11.13)$ & $71.6(11.2)$ & $\mathrm{p}=0.464$ \\
Tegner activity scale & $6.25(1.82)$ & $6.08(1.93)$ & $\mathrm{p}=0.826$ \\
Time since surgery (year) & $4.5(3.5)$ & $\mathrm{NA}$ & \\
\hline
\end{tabular}

Twelve participants in ACLR group and 12 in control group.

ACLR, anterior cruciate ligament reconstruction; NA, not applicable. 


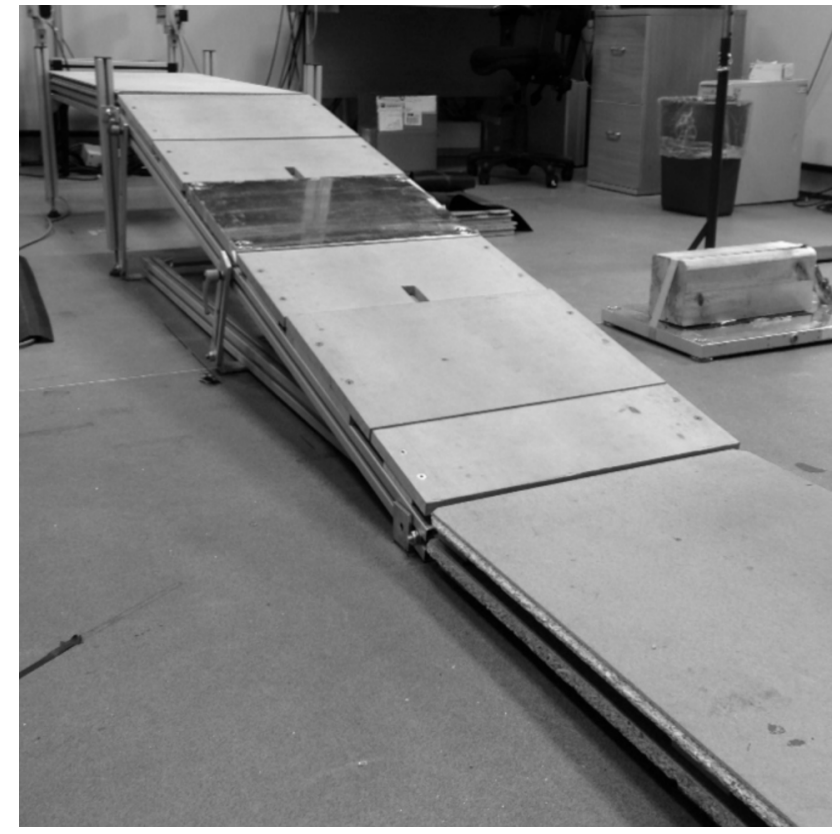

Figure 1 Steel-framed ramp covered in plywood, set an incline of $10^{\circ}$.

was constructed from a steel frame and covered with plywood, with one portable force plate embedded in the centre (figure 1). Participants were asked to walk at a self-selected pace uphill and downhill. The ramp was then removed to create a level walkway. All the participants were asked to walk at a self-selected pace and at a pace which they considered to be slow. Each task was repeated until both feet made complete contact with the middle of the force plate at least three times.

All data were time normalised to one gait cycle. The data were analysed from the stance phase of the gait cycle, when the ground reaction force reached more than $40 \mathrm{~N}$ (heel strike) to when it dropped to less than $40 \mathrm{~N}$ (toe off). A fourth order Butterworth Filter at a cut off $(12 \mathrm{~Hz})$ was used to reduce noise. Joint angles and moments were calculated from the position of the reflective markers and the ground reaction force data using a custom model written in bodybuilder software. ${ }^{23-25}$ Peak moments in the sagittal and frontal planes of the knee were extracted using MATLAB (R2013b) software.

Unpaired Student $\mathrm{t}$ tests were used to determine any significant differences in demographics between ACLR and control group. Multivariate analysis of variance test was used to calculate significant differences in all other parameters. Tukey honest significant difference approach was used to establish significance, with the $\alpha$ value set at 0.05 . All statistics were carried out using SPSS V.22.

\section{RESULTS}

Twelve ACLR participants (9 men and 3 women) and 12 controls (9 men and 3 women) were recruited for this study. There were no significant differences between the groups in age, height, weight and Tegner activity scale (table 1). The mean time since reconstruction surgery was 4 years and 6 months.

We further divided our ACLR group into two: participants that had additional cartilage, meniscus or ligament damage in their ACLR leg (ACLR+ group; 7 participants) and participants with isolated ACL injuries (ACLR- group; 5 participants). The additional knee injuries to the ACL rupture are meniscal tear (3 participants), cartilage damage (3 participants) and torn medial collateral ligament (MCL; 1 participant).

No statistically significant differences were found in peak knee adduction moment between ACLR and control participants during uphill and downhill gait, and during gait at normal and slow walking speeds on a flat surface (figure 2). Further analysis revealed that ACLR participants with meniscal tear, cartilage damage or MCL damage (ACLR+) had significantly higher knee adduction moment $(0.33 \pm 0.12 \mathrm{Nm} / \mathrm{kg} \mathrm{m})$ during gait on a flat surface at a normal walking speed compared
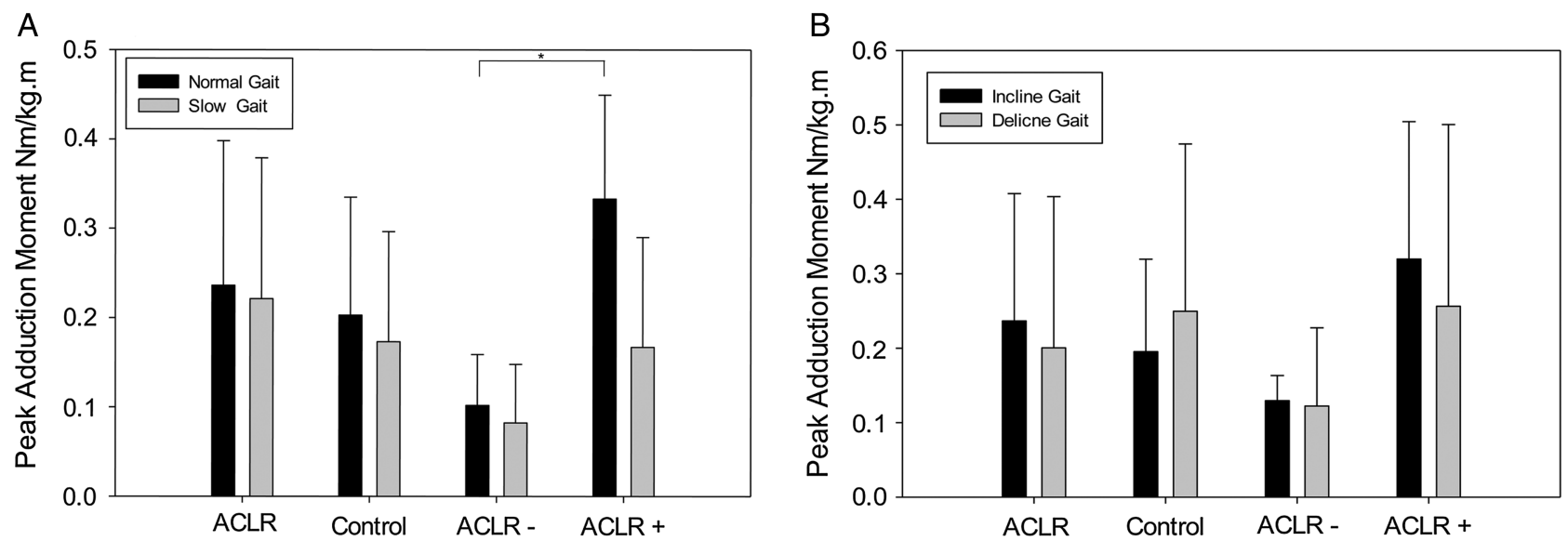

Figure 2 Peak adduction moments in (A) level walking and $(B)$ inclined walking for $A C L R, A C L R+, A C L R-$ and control group. Asterisk indicates significance $(p=0.042)$. 

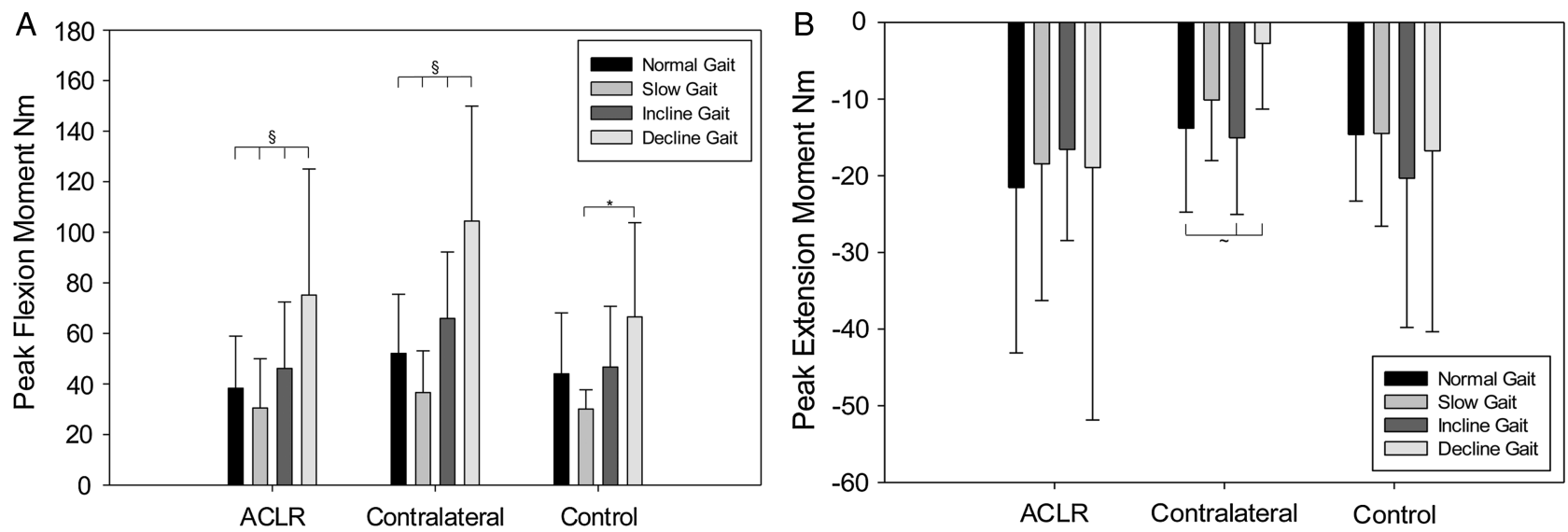

Figure 3 (A) Peak flexion moment in all activities for ACLR, contralateral and control group. §Represents decline gait to be significantly higher than all other activities $(p<0.01)$. Asterisk indicates significance, $p<0.05(B)$ Peak extension moment in all activities for ACLR, contralateral and control group. $\sim$ Represents decline gait to be significantly lower than normal and incline gait, $\mathrm{p}<0.05$.

with those participants with an isolated ACL injury (ACLR-, $0.1 \pm 0.057 \mathrm{Nm} / \mathrm{kg} \mathrm{m}$ ), $\mathrm{p}=0.042$ (figure 2).

This was not the case for data collected in the sagittal plane. There was a tendency for the contralateral (unaffected) knee of ACLR participants to show a higher mean knee flexion moment in all activities compared with ACLR affected knees and control knees (figure 3). The difference was not found to be statistically significant.

Table 2 shows that there were no significant differences in gait speed in normal or slow walking between any groups. The control group had significantly higher scores in all of the KOOS domains apart from activities of daily life compared with the ACLR group (table 3). No significant KOOS differences were seen between ACLR+ and ACLR- groups.

\section{DISCUSSION}

In the frontal plane we found no statistically significant differences between our ACLR participants and controls. However ACLR participants who had sustained associated trauma to other key knee structures (meniscal, collateral ligament and chondral damage) were observed to have a higher adduction moment during gait on a flat surface at normal walking speed when compared with participants with isolated ACLR. In the sagittal plane there was a tendency for ACLR participants to have higher peak knee flexion moment in their contralateral leg during all activities.

Previous studies that have investigated peak knee adduction moment in ACLR and matched control participants have provided mixed results. ${ }^{16-18}$ We investigated similar and more challenging gait set-ups (by altering incline), as well as the effects of differences in walking speed, in order to explore the biomechanical basis for the observation that ACL injury predisposes one to OA. Our data showed no significant differences in KAM between our ACLR participants and control participants under all conditions. This indicates that providing more challenging gait set-ups such as inclined walking, where a higher range of motion in the sagittal plane is required, does not emphasise differences between ACLR and control participants. Based on our findings, the discrepancies in previous studies appear not to be related to the difficulty of the task or differences in walking speed.

ACL injury is often accompanied by other knee injuries. ${ }^{26}$ Prevalence of associated meniscal damage and chondral lesions at the time of ACL injury can be as high as $65 \%$ and $23 \%$, respectively. ${ }^{27}$ Associated knee injuries are thought to increase the incidence of $\mathrm{OA}$ from $0-13 \%$ in isolated ACL injury to $21-48 \% .{ }^{28}$ This may be because key knee structures such as menisci prevent cartilage wear by distributing loads and functioning as shock absorbers. ${ }^{14}$ Our results suggest that

Table 2 Gait speed during normal and slow, level walking tasks

\begin{tabular}{llllll}
\hline & ACLR & Control & ACLR+ & ACLR- & p Value \\
\hline Gait normal speed & $1.17(0.13)$ & $1.20(0.11)$ & $1.18(0.15)$ & $1.16(0.11)$ & 0.940 \\
Gait slow speed & $0.76(0.13)$ & $0.75(0.11)$ & $0.78(0.16)$ & $0.74(0.09)$ & 0.885 \\
\hline
\end{tabular}

Data are mean (SD).

ACLR, anterior cruciate ligament reconstruction; ACLR+, participants with other knee injuries in their ACLR leg; ACLR-, participants with isolated ACL injuries. 
Table 3 Knee injury and Osteoarthritis Outcome Score (KOOS) with SD for each domain recorded for each group

\begin{tabular}{|c|c|c|c|c|c|}
\hline KOOS outcome & $\begin{array}{l}\text { ACLR (SD) } \\
(n=12)\end{array}$ & $\begin{array}{l}\text { Control (SD) } \\
(n=12)\end{array}$ & $\begin{array}{l}\text { ACLR+ (SD) } \\
(n=7)\end{array}$ & $\begin{array}{l}\text { ACLR- (SD) } \\
(n=5)\end{array}$ & Significant values \\
\hline Pain & $88.4(9.32)$ & $99.1(3.2)$ & $87.5(8.83)$ & $89.4(10.8)$ & $\begin{array}{l}\text { Control vs ACLR: } p=0.010 \\
\text { Control vs } A C L R(+): p=0.019\end{array}$ \\
\hline Symptoms & $83.1(11.4)$ & $98.2(3.19)$ & $85.1(12.7)$ & $80.7(10.6)$ & Control vs all other groups: $p<0.05$ \\
\hline Activities of daily life & $96.3(5.63)$ & $100(0)$ & $98(3)$ & $94.4(7.7)$ & No significant differences \\
\hline Sport and recreation & $83.8(16.9)$ & $99.6(1.4)$ & $89.1(7.4)$ & $77.4(23)$ & $\begin{array}{l}\text { Control vs ACLR: } p=0.006 \\
\text { Control vs } \operatorname{ACLR}(-): p=0.003\end{array}$ \\
\hline Knee-related QOL & $64.5(23.2)$ & $100(0)$ & $64.6(23.3)$ & $70(26.7)$ & Control vs all other groups: $p<0.05$ \\
\hline
\end{tabular}

Data are mean (SD).

ACLR, anterior cruciate ligament reconstruction; ACLR+, participants with other knee injuries in their ACLR leg; ACLR-, participants with isolated $\mathrm{ACL}$ injuries.

people with ACLR injuries with associated knee injuries experience higher knee adduction moments than people with isolated ACLR injuries.

Of the three previous studies that have looked at peak KAM in ACLR and matched control participants, Butler et $a l^{16}$ found the ACLR group to have a higher peak KAM compared with controls. This was also seen in our ACLR+ group. In the studies that followed, Webster and Feller $^{17}$ and Zabala $e t a l^{18}$ found the ACLR group to have a reduced peak knee adduction moment to controls. This was seen in our ACLR-group (figure 2). This disparity in studies may therefore be a consequence of different exclusion criteria for ACLR participants. Butler et $a l^{16}$ did not exclude ACLR participants with other knee injuries, while the other two studies excluded participants with ligament damage ${ }^{17} 18$ and also those with $>25 \%$ of menisci loss. ${ }^{18}$ We suggest that associated knee injuries are related to increased knee adduction moments in ACLR participants.

We found the difference between peak KAM in ACLR+ and ACLR - to be statistically significant only during normal gait. We expected the difference to be higher during inclined walking, as it is more challenging than level walking. Change in terrain, muscle weakness, gait deficit and balance deficit are primary risk factors for falling. ${ }^{29} 30$ This suggests that ACLR participants need to adopt a conservative gait strategy while walking on a sloped surface to ensure safety. During challenging tasks such as downhill walking, healthy participants increased their metabolic activity and implemented a conservative gait strategy to reduce the risk of falling. ${ }^{29}$ This principle may also be applied by ACLR participants to ensure safety.

In the sagittal plane, no statistically significant differences were observed between ACLR, ACLR+, ACLRand control group in peak knee flexion and extension moments. Uphill and downhill walking require greater use of quadriceps muscle than on a level walkway. ${ }^{30} 31$ Two years after an ACLR surgery, differences in quadriceps strength between limbs are no longer seen. ${ }^{29}$ All of the ACLR participants had undergone reconstruction at least 1 year before taking part in this study, with an average of 4.5 years. This indicates that all participants may have had sufficient time to restore their quadriceps strength. Although sagittal instability is thought to increase joint loads and lead to joint failure, ${ }^{14} 32$ it may not play a significant role in OA induction and progression after reconstruction.

An unexpected finding was the tendency for the contralateral knees of ACLR participants to have higher peak knee flexion and lower peak knee extension moment compared with the ACLR and control knees in all activities. This may be an adaptation to reduce loading on their ACLR knee. Patients with advanced knee OA also display this adaptation to reduce loading on their injured leg. ${ }^{33}$ Although this may present as a mechanism to slow the progression of OA, there are harmful implications associated with the contralateral leg. Weight-bearing asymmetry may induce $\mathrm{OA}$ in the contralateral leg ${ }^{34} ; 37 \%$ (24/65 female patients) showed signs of radiographic $\mathrm{OA}$ in their contralateral leg, 12 years after ACLR. ${ }^{35}$ This suggests that in this population unilateral injury changes joint function bilaterally.

In addition to our primary investigations we also investigated differences in KOOS and walking speed. There was no significant difference in KOOS or gait speed between ACLR+ and ACLR-. This indicates that high peak knee adduction moment in the injured leg does not affect our participants' pain outcome, symptoms, activities of daily life, sport and recreation, knee-related quality of life and gait speed. Therefore, patientreported outcome measures and gait speed might not provide the clinician with any information about different gait adaptations.

Mundermann et $a \vec{l}^{36}$ found a $10.2 \%$ reduction in maximum knee adduction moment when people with less severe OA reduced their walking speed from 1.2 to $0.8 \mathrm{~m} / \mathrm{s}$. However, in the current study the difference in peak knee moments between normal $(1.17 \mathrm{~m} / \mathrm{s})$ and slow gait speed $(0.76 \mathrm{~m} / \mathrm{s})$ was not statistically significant. This may be due to our small sample size.

It is important to note that different knee injuries, rehabilitation protocols and time between injury and reconstruction are all thought to influence joint moments. ${ }^{18}$ These are limitations that should be 
considered when examining the results presented here. Additionally our participants were not recruited straight after their ACLR; some participants may have had further injury or pathological changes within the joint since the reconstruction. Another limitation was our small sample size, in particular after dividing our ACLR group into ACLR+ and ACLR- groups.

The ramp was set at an incline of $10^{\circ}$ because the transition from a level to inclined walking strategy is thought to be around $5.5^{\circ 31}$ and after an incline of $10^{\circ}$ no kinematic differences are seen in healthy participants. ${ }^{31}$

With regard to Vicon Motion capture system, different skin marker placement and skin motion artefacts are thought to increase error. ${ }^{6}$ We tried to reduce the effects of different skin marker placement by having only one researcher place all markers on each individual. In addition we used a model that used clusters to reduce the effects of skin motion. ${ }^{25}$

\section{CONCLUSION}

In conclusion, this study found no significant differences in peak moments in the frontal and sagittal planes during level and inclined walking for ACLR compared with control participants. However, we noted that individuals who have other knee injuries associated with their ACLR knee exhibit higher peak adduction moments during level walking at their normal speed. This suggests that injuries to other key knee structures may play a bigger part in inducing OA than ACL injury alone, although this requires further investigation with a larger sample size. Our data also suggest that the contralateral knee appears to be functioning in such a way to reduce high moments in the ACLR knees, which may be relevant in the risk of $\mathrm{OA}$ development in both knees. These findings warrant a longitudinal study comparing the knee adduction moment between isolated ACLR injury and ACLR with additional knee injuries and the prevalence of premature OA.

Acknowledgements The authors acknowledge support from Mr R Weinert-Aplin for the facilitation of the instrumented inclined walkway and conversion of the output data. He was also involved in designing the data collection tool.

Contributors RKV carried out data collection, statistical analysis, analysis of the data produced. He also drafted, revised the manuscript and is the guarantor. LDD attained ethical approval, determined the methodology, helped with data analysis, drafted and revised the paper. DN helped with data collection and drafted and revised the paper. AHM monitored the data collection for the whole trial, provided the data collection tools, drafted and revised the paper.

Funding The authors acknowledge the support from the Medical Engineering Solutions in Osteoarthritis Centre of Excellence funded by the Wellcome Trust and EPSRC.

\section{Competing interests None.}

Ethics approval Imperial College Research Ethics Committee.

Provenance and peer review Not commissioned; externally peer reviewed.

Data sharing No additional data are available.
Open Access This is an Open Access article distributed in accordance with the terms of the Creative Commons Attribution (CC BY 3.0) license, which permits others to distribute, remix, adapt and build upon this work, for commercial use, provided the original work is properly cited. See: http:// creativecommons.org/licenses/by/3.0/

\section{REFERENCES}

1. Lyman $S$, Koulouvaris $P$, Sherman $S$, et al. Epidemiology of anterior cruciate ligament reconstruction: trends, readmissions, and subsequent knee surgery. J Bone Joint Surg Am 2009;91:2321-8.

2. Matsumoto $H$, Suda $Y$, Otani $T$, et al. Roles of the anterior cruciate ligament and the medial collateral ligament in preventing valgus instability. J Orthop Sci 2001;6:28-32.

3. Lohmander LS, Englund PM, Dahl LL, et al. The long-term consequence of anterior cruciate ligament and meniscus injuries: osteoarthritis. Am J Sports Med 2007;35:1756-69.

4. Porat $\mathrm{A}$, Roos EM, Roos $\mathrm{H}$. High prevalence of osteoarthritis 14 years after an anterior cruciate ligament tear in male soccer players: a study of radiographic and patient relevant outcomes. Ann Rheum Dis 2004;63:269-73.

5. Andriacchi TP, Mündermann A. The role of ambulatory mechanics in the initiation and progression of knee osteoarthritis. Curr Opin Rheumatol 2006;18:514-18.

6. Stergiou N, Ristanis S, Moraiti C, et al. Tibial rotation in anterior

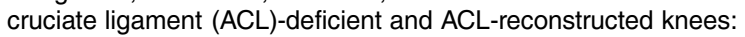
a theoretical proposition for the development of osteoarthritis. Sports Med 2007;37:601-13.

7. Andriacchi TP, Mündermann $A$, Smith $R L$, et al. A framework for the in vivo pathomechanics of osteoarthritis at the knee. Ann Biomed Eng 2004;32:447-57.

8. Hart JM, Ko JW, Konold T, et al. Sagittal plane knee joint moments following anterior cruciate ligament injury and reconstruction: a systematic review. Clin Biomech 2010;25:277-83.

9. Georgoulis AD, Papadonikolakis A, Papageorgiou CD, et al. Three-dimensional tibiofemoral kinematics of the anterior cruciate ligament-deficient and reconstructed knee during walking. $A m \mathrm{~J}$ Sports Med 2003;31:75-9.

10. Noyes FR, Schipplein OD, Andriacchi TP, et al. The anterior cruciate ligament-deficient knee with varus alignment. An analysis of gait adaptations and dynamic joint loadings. Am J Sports Med 1992;20:707-16.

11. Gokeler A, Benjaminse A, van Eck CF, et al. Return of normal gait as an outcome measurement in ACL reconstructed patients. A systematic review. Int J Sports Phys Ther 2013;8:441-51.

12. Berchuck M, Andriacchi TP, Bach BR, et al. Gait adaptations by patients who have a deficient anterior cruciate ligament. J Bone Joint Surg Am 1990;72:871-7.

13. Urbach D, Nebelung W, Becker R, et al. Effects of reconstruction of the anterior cruciate ligament on voluntary activation of quadriceps femoris a prospective twitch interpolation study. J Bone Joint Surg Br 2001;83:1104-10.

14. Roos EM. Joint injury causes knee osteoarthritis in young adults. Curr Opin Rheumatol 2005;17:195-200.

15. Papageorgiou CD, Gil JE, Kanamori A, et al. The biomechanical interdependence between the anterior cruciate ligament replacement graft and the medial meniscus. Am J Sports Med 2001;29:226-31.

16. Butler RJ, Minick KI, Ferber R, et al. Gait mechanics after ACL reconstruction: implications for the early onset of knee osteoarthritis. Br J Sports Med 2009;43:366-70.

17. Webster KE, Feller JA. The knee adduction moment in hamstring and patellar tendon anterior cruciate ligament reconstructed knees. Knee Surg Sports Traumatol Arthrosc 2012;20:2214-19.

18. Zabala ME, Favre J, Scanlan SF, et al. Three-dimensional knee moments of ACL reconstructed and control subjects during gait, stair ascent, and stair descent. J Biomech 2013;46:515-20.

19. Miyazaki T, Wada M, Kawahara $\mathrm{H}$, et al. Dynamic load at baseline can predict radiographic disease progression in medial compartment knee osteoarthritis. Ann Rheum Dis 2002;61:617-22.

20. von Elm E, Altman DG, Egger M, et al.; STROBE Initiative. The Strengthening the Reporting of Observational Studies in Epidemiology (STROBE) statement: guidelines for reporting observational studies. J Clin Epidemiol 2008;61:344-9.

21. Roos EM, Roos HP, Lohmander LS, et al. Knee Injury and Osteoarthritis Outcome Score (KOOS) - development of a self-administered outcome measure. J Orthop Sports Phys Ther 1998;28:88-96.

22. Tegner Y, Lysholm J. Rating systems in the evaluation of knee ligament injuries. Clin Orthop 1985;198:43-9. 
23. Cleather DJ, Bull AM. Influence of inverse dynamics methods on the calculation of inter-segmental moments in vertical jumping and weightlifting. Biomed Eng Online 2010;9:74

24. Cleather DI, Bull A. Lower-extremity musculoskeletal geometry affects the calculation of patellofemoral forces in vertical jumping and weightlifting. Proc Inst Mech Eng H 2010;224:1073-83.

25. Hope N, Duffell LD, McGregor AH. Validation of a new model to calculate joint kinematics during gait. European Society of Biomechanics Conference Proceedings 1-7-2012.

26. Claes S, Hermie L, Verdonk R, et al. Is osteoarthritis an inevitable consequence of anterior cruciate ligament reconstruction? A meta-analysis. Knee Surg Sports Traumatol Arthrosc 2013;21:1967-76.

27. Louboutin H, Debarge R, Richou J, et al. Osteoarthritis in patients with anterior cruciate ligament rupture: a review of risk factors. Knee 2009;16:239-44.

28. Oiestad BE, Engebretsen L, Storheim K, et al. Knee osteoarthritis after anterior cruciate ligament injury: a systematic review. Am J Sports Med 2009;37:1434-43.

29. Monsch ED, Franz CO, Dean JC. The effects of gait strategy on metabolic rate and indicators of stability during downhill walking. J Biomech 2012;45:1928-33.
30. Mizner RL, Snyder-Mackler L. Altered loading during walking and sit-to-stand is affected by quadriceps weakness after total knee arthroplasty. J Orthop Res 2005;23:1083-90.

31. Lay AN, Hass CJ, Gregor RJ. The effects of sloped surfaces on locomotion: a kinematic and kinetic analysis. J Biomech 2006;39:1621-8.

32. Gottschall JS, Nichols TR. Neuromuscular strategies for the transitions between level and hill surfaces during walking. Philos Trans $R$ Soc Lond B Biol Sci 2011;366:1565-79.

33. Creaby MW, Bennell KL, Hunt MA. Gait differs between unilateral and bilateral knee osteoarthritis. Arch Phys Med Rehabil 2012;93:822-7.

34. Christiansen CL, Stevens-Lapsley JE. Weight-bearing asymmetry in relation to 378 measures of impairment and functional mobility for people with knee osteoarthritis. Arch Phys Med Rehabil 2010;91:1524-8.

35. Lohmander LS, Ostenberg A, Englund M, et al. High prevalence of knee osteoarthritis, pain, and functional limitations in female soccer players twelve years after anterior cruciate ligament injury. Arthritis Rheum 2004;50:3145-52.

36. Mündermann A, Dyrby CO, Hurwitz DE, et al. Potential strategies to reduce medial compartment loading in patients with knee osteoarthritis of varying severity: reduced walking speed. Arthritis Rheum 2004;50:1172-8. 